\title{
Re: Controversies in Odontogenic Tumours
}

Review

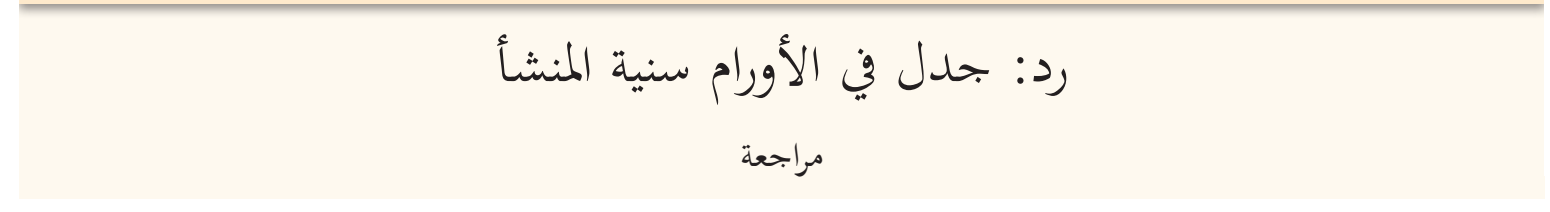

Dear Editor,

We read with great interest the recent review article by Siwach et al. published in the August 2017 issue of SQUMJ. ${ }^{1}$ We congratulate the authors on their elaborative narrative review of the controversies regarding various tumours of odontogenic origin. We would like to further contribute by adding an interesting odontogenic tumour to the debate-adenoid ameloblastomas with dentinoid. First reported by Slabbert et al., this tumour shows histopathological features of both an ameloblastoma and an adenomatoid odontogenic tumour (AOT), along with hard tissue formation (i.e. dentin or dentinoid-like material). ${ }^{2}$ While ameloblastic elements such as epithelial whorls, duct-like structures and occasional signs of induction cover a major portion of the tumour, AOT-like areas are also visually evident, indicating a composite odontogenic tumour. ${ }^{2}$

Initially known as dentinoameloblastomas, the suggestion that these tumours were the result of the hybridisation of an ameloblastoma and AOT with fibrous separation resulted in significant controversy, as numerous cases revealed duct-like structures with bordering cells displaying subnuclear vacuoles. ${ }^{2,3}$ Subsequently, Loyola et al. described a similar neoplasm with the potential for recurrence and suggested the term 'adenoid ameloblastoma with dentinoid' for such lesions. ${ }^{3}$ To date, adenoid ameloblastomas have not yet been included by the World Health Organization (WHO) in the histological classification of odontogenic tumours. ${ }^{4}$ The exclusion of this entity is interesting as other tumours with comparatively fewer documented cases, such as sclerosing odontogenic carcinomas, have nevertheless been incorporated in the latest WHO classification of odontogenic tumours. ${ }^{4}$

While the nature of the hard tissue in these tumours is unknown, ectomesenchymal cells showing odontoblastic differentiation can be completely absent. ${ }^{5}$ According to Sonone et al., the formation of dysplastic dentin in these tumours may be the result of a metaplastic process rather than the currently accepted theory of epithelial-ectomesenchymal interaction. ${ }^{5}$ This new hypothesis is further supported by the expression of certain genes by ameloblastic epithelial cells usually present in ectomesenchymal cells, thereby resulting in the conversion and co-expression of a mesenchymal phenotype. ${ }^{6}$ Further studies with more detailed molecular profiling are needed to determine whether the hard tissue in adenoid ameloblastoma is the result of a metaplastic process or a true inductive phenomenon.

The co-occurrence of multiple phenotypes of ameloblastoma within the same lesion is not uncommon. ${ }^{4}$ In general, the diverse histological hybridisation of subtypes of solid and multicystic ameloblastoma does not impact the clinical behaviour of the lesion. However, substantial evidence indicates that adenoid ameloblastomas are locally aggressive. ${ }^{3}$ Adenoid ameloblastomas can present with hypercellularity and atypia, thus posing a challenge in distinguishing these lesions from ameloblastic carcinomas. Nevertheless, features of loss of ameloblastic differentiation, basilar hyperplasia, necrosis and vascular/perineural invasion can assist in differentiating a true ameloblastic carcinoma from an adenoid ameloblastoma. ${ }^{3}$

\author{
*Divya Gopinath ${ }^{1}$ and Rohit K. Menon ${ }^{2}$ \\ ${ }^{1}$ Department of Oral Rehabilitation, Faculty of Dentistry, University of Hong Kong, Hong Kong; ${ }^{2}$ Department of Prosthodontics, \\ International Medical University, Kuala Lumpur, Malaysia \\ "Corresponding Author's e-mail: drdivyakmenon@gmail.com
}




\section{References}

1. Siwach P, Joy T, Tupkari J, Thakur A. Controversies in odontogenic tumours: Review. Sultan Qaboos Univ Med J 2017; 17:e268-76. doi: 10.18295/squmj.2017.17.03.003.

2. Slabbert H, Altini M, Crooks J, Uys P. Ameloblastoma with dentinoid induction: Dentinoameloblastoma. J Oral Pathol Med 1992; 21:46-8. doi: 10.1111/j.1600-0714.1992.tb00969.x.

3. Loyola AM, Cardoso SV, de Faria PR, Servato JP, Eisenberg AL, Dias FL, et al. Adenoid ameloblastoma: Clinicopathologic description of five cases and systematic review of the current knowledge. Oral Surg Oral Med Oral Pathol Oral Radiol 2015; 120:368-77. doi: 10.1016/j. oooo.2015.05.011.

4. El-Naggar AK, Chan JK, Grandis JR, Takata T, Slootweg PJ, Eds. WHO Classification of Head and Neck Tumours, 4th ed. Lyon, France: IARC Press, 2017.

5. Sonone A, Hande A, Chaudhary M, Bonde R, Sheorain A, Agni N. Adenoid ameloblastoma with dentinoid and ghost cells: A composite odontogenic tumour - A rare case report and review of the literature. Oral Surg 2011; 4:77-81. doi: 10.1111/j.1752-248X.2010.01109.x.

6. Papagerakis P, Peuchmaur M, Hotton D, Ferkdadji L, Delmas P, Sasaki S, et al. Aberrant gene expression in epithelial cells of mixed odontogenic tumors. J Dent Res 1999; 78:20-30. doi: 10.1177/00220345990780010201.

\section{Response from the Authors}

\section{Dear Reader,}

Thank you very much for reading our article and for your suggestion to include adenoid ameloblastomas with dentinoid to the current debate regarding odontogenic tumours. We did not include this entity in our review article because it was not incorporated in the 2017 World Health Organization classification of odontogenic tumours. ${ }^{1,2}$ Considerable variations in the histopathological presentation of epithelial odontogenic tumours can sometimes be confusing and increase the chance of misdiagnosis. ${ }^{3}$ An adenoid ameloblastoma with dentinoid is considered a hybrid tumour of the odontogenic apparatus in that it demonstrates the histopathological characteristics of two or more previously recognised odontogenic tumours and/or cysts. ${ }^{4}$ The exact cause for this is not known. Odontogenic tumours originate from the odontogenic epithelium, which has the potential for diverse differentiation and complex inductive interactions. Therefore, it is possible that the pluripotent odontogenic epithelium can induce the formation of different histopathological patterns within the same tumour. ${ }^{5}$

Only a few cases have been reported of tumours with histopathological features of both an ameloblastoma and an adenomatoid odontogenic tumour (AOT), along with hard tissue formation. ${ }^{6}$ Of these, almost half of the cases were initially diagnosed as ameloblastomas and treated via resection, while the others were believed to be AOTs and were consequently undertreated, leading to the recurrence of the lesion. In such cases, the AOT-like areas predominated, overshadowing the ameloblastomatous areas and leading to a benign diagnosis and conservative treatment. ${ }^{6}$ Therefore, a thorough examination of the biopsy sample is mandatory to rule out the existence of another lesion; moreover, it is better that such hybrid lesions be treated with the more aggressive lesion in mind. Similarly, Ide et al. reported a lesion initially diagnosed as a globulomaxillary cyst, subsequently identified as an AOT upon histopathological analysis and retrospectively confirmed to be consistent with an adenoid ameloblastoma with dentinoid after the lesion had recurred four times over an 11-year period. ${ }^{3}$ According to the authors, most lesions reported in the literature as recurrent AOTs are almost certainly adenoid ameloblastomas with dentinoid. ${ }^{3}$

Whether hybrid tumours should be considered to be new entities, subtypes of two separate odontogenic lesions or solely histopathological variations of established entities is yet to be concluded. ${ }^{4,5}$ However, while such hybrid histopathological appearances illustrate the differentiation potential of the odontogenic epithelium and ectomesenchyme along with their complex inductive interactions, a detailed subdivision is meaningless in clinical practice. ${ }^{4}$ Furthermore, considering these hybrid lesions to be new entities may further complicate the already long list of odontogenic tumours and cause confusion with regards to widely accepted terminology. ${ }^{2}$ Nevertheless, it is important that oral pathologists bear knowledge of such hybrid lesions in mind so that they can aid the operating surgeon when decided on the best treatment plan for affected patients.

"Pooja Siwach, Tabita Joy, Jagdish Tupkari, Arush Thakur

Department of Oral Pathology \& Microbiology, Government Dental College \& Hospital, Mumbai, Maharashtra, India

"Corresponding Author's e-mail: poojasiwach127@gmail.com 


\section{References}

1. Siwach P, Joy T, Tupkari J, Thakur A. Controversies in odontogenic tumours: Review. Sultan Qaboos Univ Med J 2017; 17:e268-76. doi: 10.18295/squmj.2017.17.03.003.

2. El-Naggar AK, Chan JK, Grandis JR, Takata T, Slootweg PJ, Eds. WHO Classification of Head and Neck Tumours, 4th ed. Lyon, France: IARC Press, 2017.

3. Ide F, Mishima K, Saito I, Kusama K. Diagnostically challenging epithelial odontogenic tumors: A selective review of 7 jawbone lesions. Head Neck Pathol 2009; 3:18-26. doi: 10.1007/s12105-009-0107-4.

4. Ide F, Horie N, Shimoyama T, Sakashita H, Kusama K. So-called hybrid odontogenic tumors: Do they really exist? Oral Med Pathol 2001; 6:13-21. doi: 10.3353/omp.6.13.

5. Choudhari SK, Gadbail AR. Hybrid odontogenic tumors: A controversy. Pathol Oncol Res 2015; 21:501-2. doi: 10.1007/s12253-014-9844-0.

6. Saxena K, Jose M, Chatra LK, Sequiera J. Adenoid ameloblastoma with dentinoid. J Oral Maxillofac Pathol 2012; 16:272-6. doi: 10.4103/0973029X.99088. 